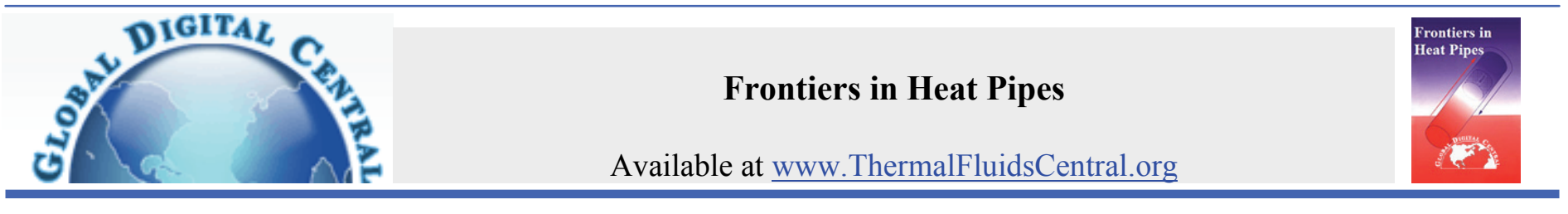

\title{
SOME CHARACTERISTICS OF HEAT PIPE WICKS CREATED BY A SIMPLE ELECTROCHEMICAL PROCESS
}

\author{
Rumen Kirilov ${ }^{*}$, Petko Stefchev \\ Central Laboratory of Solar Energy and New Energy Sources at Bulgarian Academy of Sciences, 72 "Tzarigradsko chaussee" blvd., 1784, Sofia, \\ Bulgaria
}

\begin{abstract}
Evaporation of pure water out of $\mathrm{Cu}$ micro-porous dendrite surface layers is investigated. These layers are deposited on $\mathrm{Cu}$ substrates using twostage electrochemical process. The basic microstructure obtained in the first stage using current density of $40 \mathrm{~A} / \mathrm{dm} 2 \mathrm{was}$ further strengthened by continuing electrochemical deposition employing much lower current density of $2.5 \mathrm{~A} / \mathrm{dm} 2$. Scanning Electron Microscopy (SEM) shows that the layers have porous dendrite structure. Samples were submitted to a water-thermal treatment to make them hydrophilic. Their evaporation capability was tested using a simple setup. Tests for determining of the liquid permeability and porosity are also carried out.
\end{abstract}

Keywords: Electro deposition, Heat Pipe, Capillary Wick, Microstructure

\section{INTRODUCTION}

Fluidic-based cooling/heating solutions have commonly been incorporated in units using traditional large-scale air heat dissipation including pumped liquid based cooling/heating. Such systems have suffered from inefficiencies and exhibit limited performance characteristics. The use of heat pipes is an alternative.

A heat pipe can create a near isothermal heat sink/source and minimize the overall thermal resistance increasing the efficiency of the system and allowing it to function in lower temperature differences.

The main components of a heat pipe are a wick structure element and a working fluid. The heat pipe has three sections: evaporator, adiabatic or transport, and condenser.

In the current investigation, an electrodeposition method for fabricating wicks on copper surfaces is used. This method can be applied to create dendritic copper wicks with controllable porosity and pore size (Nikolić et al., 2006; Kim et al., 2006). The procedure can be modified to yield a wick which has a desired porosity, minimum meniscus radius, and thermal conductivity by changing the acidity, salt content, and current density of the electroforming bath.

\section{ELECTROCHEMICAL DEPOSITION}

The $\mathrm{Cu}$ micro-porous layers of different thicknesses are deposited onto $\mathrm{Cu}$ substrates of 25 by $100 \mathrm{~mm}$ area, and $0.5 \mathrm{~mm}$ thickness and the electroplated surface is 25 by $70 \mathrm{~mm}$. The substrates functioning as a cathode during the electrochemical deposition process are situated perpendicular and parallel to an insoluble $\mathrm{Pb}$ anode of 25 by $100 \mathrm{~mm}$. The coating on back side of the cathode is eliminated and thus an almost uniform electrochemical deposition of $\mathrm{Cu}$ atoms onto the working surface is achieved. During the electrochemical deposition, the $\mathrm{Cu}$ cathode and the anode, situated at a distance of
$8 \mathrm{~cm}$ are immersed in the electrolyte solution of sulfuric acid and copper sulfate. The cathode and the anode are connected to an external DC power supply, controlled by a desktop PC, to adjust the supplied current density and monitor the disposition time. The composition of the electrolyte is $0.6 \mathrm{~mol} / 1 \mathrm{CuSO}_{4}$ and $0.6 \mathrm{~mol} / 1 \mathrm{H}_{2} \mathrm{SO}_{4}$ and is working at room temperature. The change in electrolyte concentration with the plating time is negligible.

The electrochemical deposition of the copper micro-porous surface layers is carried out in two successive stages using the same electrolyte solution. The basic micro-structure, Sample A, obtained in the first stage using current density of $40 \mathrm{~A} / \mathrm{dm}^{2}$ for 60 seconds is strengthened by continuing electrochemical deposition using much lower current density of $2.5 \mathrm{~A} / \mathrm{dm}^{2}$ for 60 minutes. In this way the powder like coatings with low mechanical stability, characteristic for the first stage, transform to mechanically stable structure. The test Sample B is produced by repeating of the same electrochemical procedure. The surface morphology of the coatings was examined by scanning electron microscopy (SEM) using a JEM-200CX electron microscope.

\subsection{Experimental results}

When the electric current is supplied to the electrochemical deposition cell, the $\mathrm{Cu}^{+2}$ and $\mathrm{H}^{+}$ions in the electrolyte solution undergo two simultaneous reduction reactions at the cathode:

$$
\begin{aligned}
& \mathrm{Cu}^{+2}+2 \mathrm{e}^{-}=\mathrm{Cu} \\
& 2 \mathrm{H}^{+}+2 \mathrm{e}^{-}=\mathrm{H}_{2} \uparrow
\end{aligned}
$$

The thickness and the basic dendrite micro-structure of the layers are established in the first stage using a current density of 40 $\mathrm{A} / \mathrm{dm}^{2}$ for $60 \mathrm{~s}$. During this stage, the deposited copper atoms on the

\footnotetext{
*Corresponding author.Email:rumenkirilov@abv.bg
} 
cathode surface nucleate and grow rapidly in the form of fine and dense dendrites that surround open, circular macro-pores. The hydrogen molecules evolving at the cathode form tiny bubbles that subsequently coalesce into dense streams of rising large bubbles. The initially deposited micro-structures with high volume porosity and orderly arranged macro-pores are very delicate for handling, easily chipped and cracked. To strengthen these micro-structures, electrochemical deposition continues at much lower current density for additional 60 minutes to produce sample A. During this stage, the rate of deposition of the $\mathrm{Cu}$ atoms on the cathode is low, negligibly changing the thickness of the $\mathrm{Cu}$ dendrite surface layer. The $\mathrm{Cu}$ atoms partially fill out the dendrites structure and some of the macropores.

On Fig.1 SEM micrograph of sample A at a magnification of 78 is presented. Dendrites and large pores between them can be clearly seen. Fig. 2 presents SEM micrograph of the same sample at a magnification of 406. A dendrite with small pores into it and a large pore nearby (upper right) can be seen.

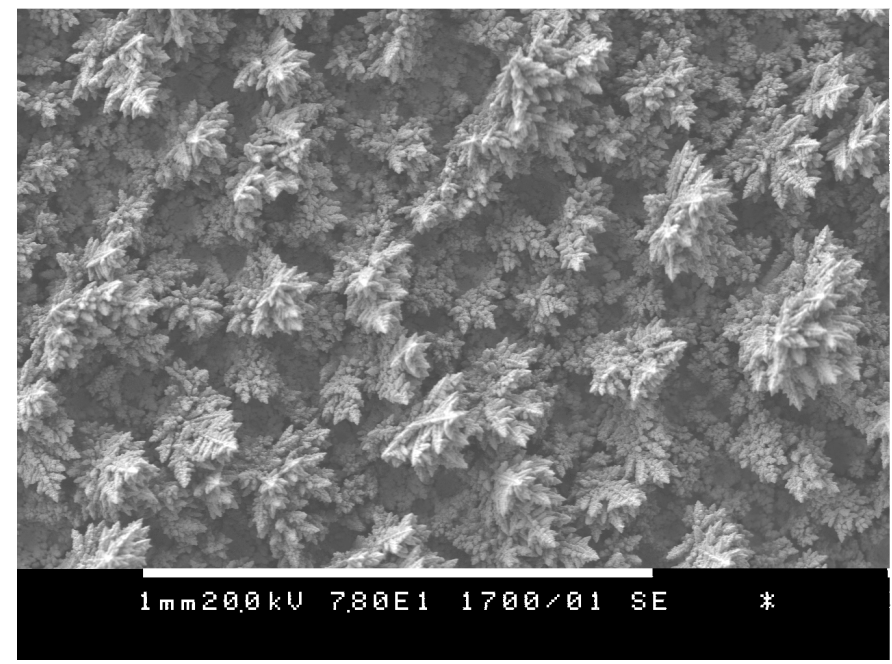

Fig. 1 SEM micrograph of sample A at a magnification of 78

SEM micrograph of the sample B presents the same structure but the dendrites are almost twice higher.

Conventional homogenous wicks with small pores may have a small characteristic capillary radius and a high effective thermal conductivity, but have low liquid permeability. At high heat fluxes, the working fluid in even small pores starts to nucleate, and due to a fine pore structure the formed vapor cannot leave the evaporator and vapor pockets start to form and to prevent rewetting of the evaporator.

Wicks with two characteristic pore sizes like in our case were able to remove much more heat at the same superheat than homogenous wicks. The two pore sizes wicks yield improved performance through increased capillary pressure and vapor permeability (El-Genk et al., 2010).

\section{HYDROPHILIC TREATMENT}

As is well known, pure copper is hydrophobic and is not suitable for a heat pipe wick (Dunn et al., 1978). Very often water treatment is used to make copper wicks hydrophilic (Min \& Webb 2002). We try the oxidation method described in (Dunn et al., 1978) without of much success. The next attempt was to immerse the lower part of the sample plate (part covered with wick) into water and left upper part dry. On the dry part we apply some heat flux (around 3W). After a few days the lower part becomes mat black (if dry) and presents excellent hydrophilic properties. This process is very slow but is "automatic" - does not need labor during preparation.

\section{THERMAL TEST SETUP}

Many investigators study wick performance by building actual heat pipes and subjecting them to heat loads while measuring external temperatures (Faghri 1995; Peterson 1994). This method has the advantage of simplicity, but is limited by what can be inferred from the external measurements, making it difficult to distinguish between cause and effect. Several investigators have attempted to improve upon the external measurements by placing the wick in an environmentally controlled test chamber (Iverson et al., 2007).

Our simple test setup as presented in Fig. 3 consists of a water pot, heater, sample plate, digital thermometer with thermocouple, power supply and a desktop computer.

Wick samples were prepared on one side of a 25 by 100 by 0.5 $\mathrm{mm}$ copper plate. The upper part of the plate was left clear (without

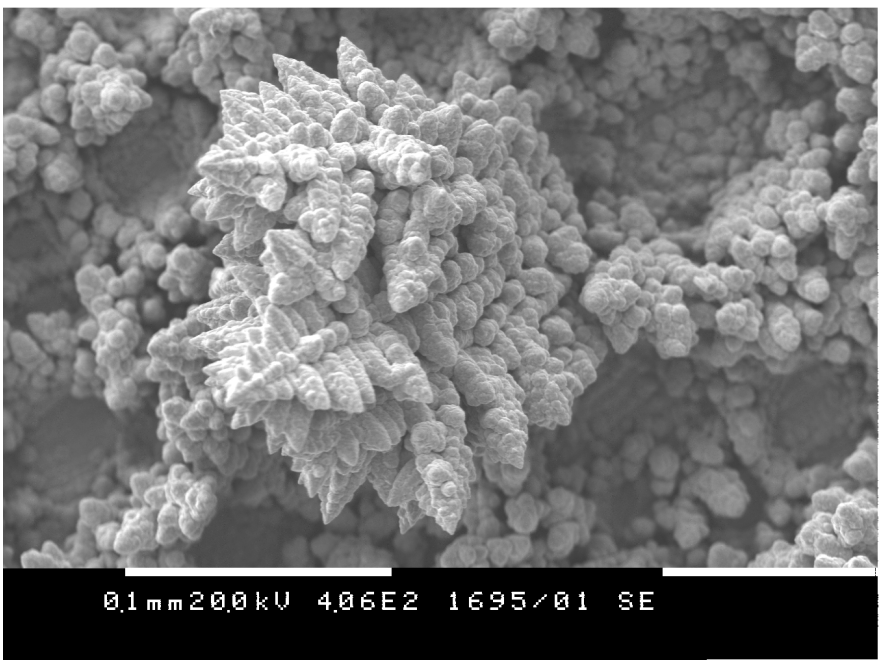

Fig. 2 SEM micrograph of the same sample A at a magnification of 406

wick) to ensure enough place for heater mounting. A power bipolar transistor in TO3 case was used as a heating element.

A steel plate ( $5 \mathrm{~mm}$ thick) was used on the back side of the copper plate to serve as a flat base and ensure proper tight contact between the case of the power transistor and the copper plate.

A proper heat conducting wax was used between case of the transistor and copper plate. Additionally a thermocouple of type $\mathrm{K}$ was mounted between power transistor case and copper plate. The lower edge of the copper plate with wick was immersed in a pot with deionized water. The diameter of the pot was large enough $(18 \mathrm{~cm})$ to

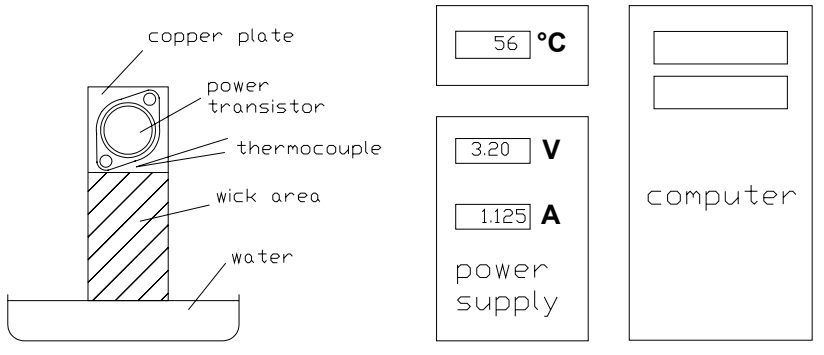

Fig. 3 Thermal test setup 
ensure constant water level during the experiment. The temperature was $15^{\circ} \mathrm{C}$ at relative humidity of $60 \%$.

\subsection{Test method}

Initially both samples were heated periodically (10 hours per day, seven days continuously) at a power of $3 \mathrm{~W}$ to ensure maximum possible wetting. After that a real test procedure starts, as follows.

The power of the heating element was changed by increasing the voltage applied to the power transistor starting with $3 \mathrm{~V}$ with steps of $0.2 \mathrm{~V}$.

After making a voltage step we ensure a period of 15 minutes to let system reaching a quazi-equilibrium state and measure the temperature and current. Forward steps were canceled when the temperature goes above $100^{\circ} \mathrm{C}$ in order to prevent transistor from overheating. Then backward steps of $0.2 \mathrm{~V}$ start. The backward steps are canceled when the supply voltage reaches $3 \mathrm{~V}$. This procedure was repeated five times per sample.

Finally we let the samples to dry for a week and repeat the above procedure of step heating and cooling keeping the wick dry. This let us to determine the net heat losses of the heating part and the back side (without wick) of the copper plate.

If subtracting the power, dissipated by the dry plate from the one, dissipated by the wet plate leads to not accurate results due to higher temperature of the dry plate at the same power. This higher temperature leads to more intensive convection than in the case of the wet plate. Looking back to the classical law:

$$
q=U^{*} F^{*}(T p-T a)
$$

and assuming that $U$ is constant at both cases (which is not correct really), we can assume that dissipated power is proportional to temperature difference only.

In the above Eq. (1) $q$ is the power dissipated from sample, $U$ is convection heat transfer coefficient, $T p$ is the mean plate temperature and $\mathrm{Ta}$ is the ambient temperature.

Our experimental data for sample A show that the mean temperature of the dry plate is with $5^{\circ} \mathrm{C}$ higher than the temperature of the wetted plate. Recalculating the power dissipated from dry sample A using equation (1) and subtracting from the power dissipated from the wet sample A at the same temperature we can take more realistic value for the water evaporated from this sample.

The same correction procedure as above for sample A was applied to sample B.

Finally, as a result, the calculated water evaporated from sample $\mathrm{A}$ is $10 \%$ higher than the one shown in Fig.4. Respectively, in the case of Sample B, the calculated water evaporated from sample B is $7 \%$ higher than that shown in Fig.5.

A small program written in assembly which uses parallel port as output and input is created to control digital thermometer, power supply unit, store results and ensures exact time between measurements.

\section{RESULTS AND DISCUSSIONS}

Figures 4 and 5 show the experimental results for heat fluxes applied to sample A and sample B, respectively. The $\mathrm{X}$ axes for figures 4, 5, 6 and 7 present values of the voltage applied to power transistor.

Upper lines present whole power dissipated by the corresponding sample including convection and radiation from plate, power transistor and evaporated water. Lower lines present power dissipated only by evaporation of water from the samples. This power is calculated by subtracting the energy dissipated from dry sample from energy dissipated from wet sample at one and the same temperature, as it was described above. It is seen that sample B can withdraw more power than sample A. This is caused mainly by the larger wick evaporation surface of B sample and its bigger thickness which allows the heat to be transferred along the plate in opposite direction of the water flow. These tests are not intended to show the maximum possible heat flux density and we have not reached conditions where a part of the wick starts to dry due to the limitation in permeability of the wick.

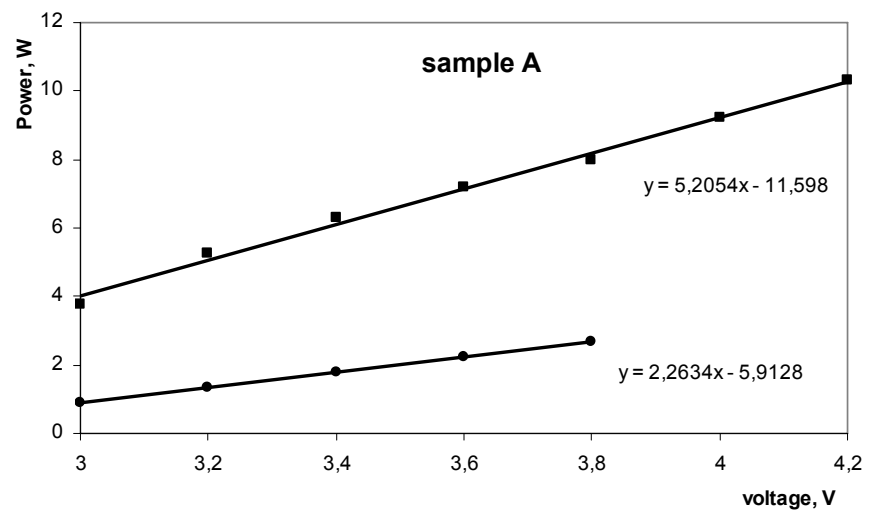

Fig. 4 Dependence of power dissipated by sample A versus voltage applied

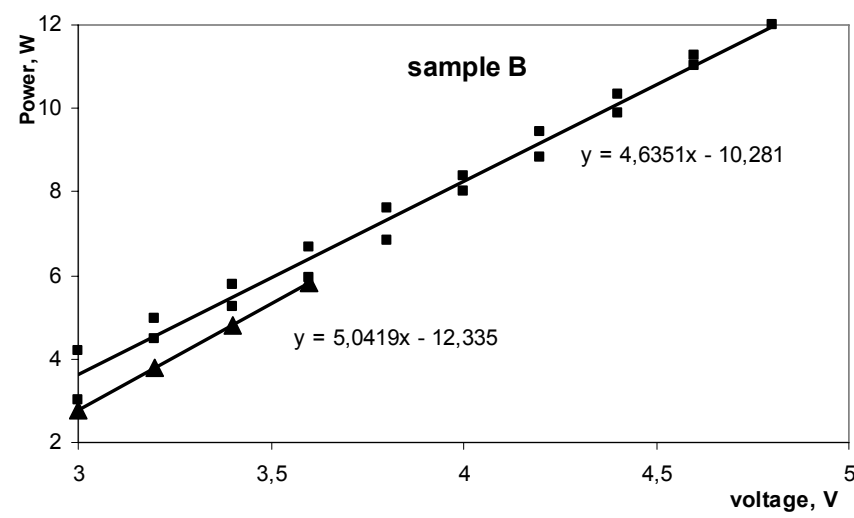

Fig. 5 Dependence of power dissipated by sample B versus voltage applied

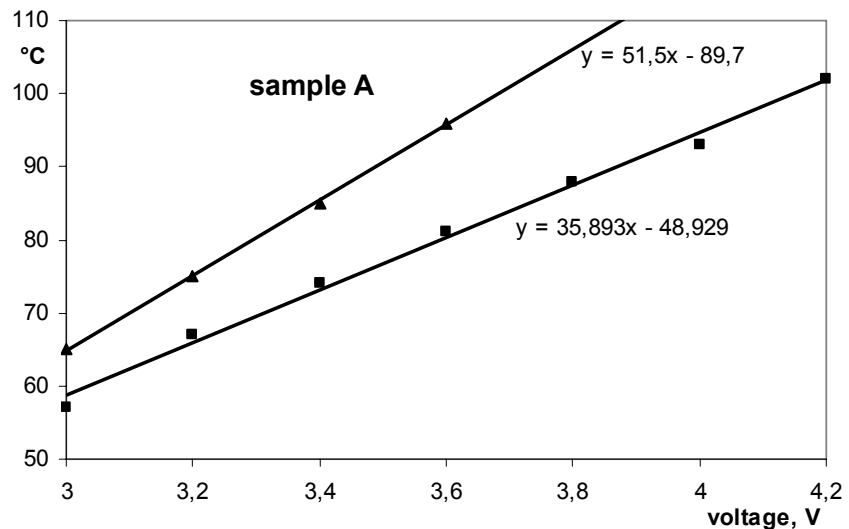

Fig. 6 Dependence of temperatures versus voltage for sample A

Temperatures achieved during tests are presented on Fig. 6 and Fig.7.

Upper lines present temperature of the interface power transistor/copper plate when the wick is dry. The lower lines present temperatures at the same points as above but with wick in operation.

A test for porosity was carried out, as follows: after finishing of thermal test we cut the part of copper plates covered with wicks, 
measure the size with an accuracy of $0.1 \mathrm{~mm}$ and weight them on a laboratory weights with an accuracy of $0.0001 \mathrm{~g}$. Then we let the samples to dry for a long period of time at room temperature and weight them again.

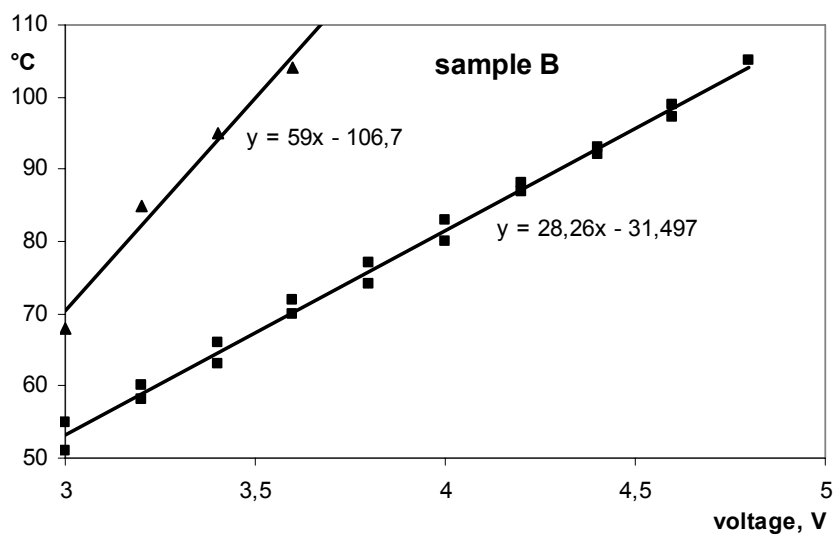

Fig. 7 Dependence of temperatures versus voltage for sample B

From the weight of the wet samples we subtract the weight of the dry samples and from the weight of the dry samples we subtract the weight of the copper plate. As a result we have the weight of the wicks plus the weight of the pores filled with water. Knowing the density of the water $\left(998 \mathrm{~kg} / \mathrm{m}^{3}\right.$ at $\left.20^{\circ} \mathrm{C}\right)$ and assuming that during the working period all air was withdrawn out of the pores one can calculate the volume of the pores. For sample A porosity was estimated as $64 \%$ and for the sample B - $83 \%$.

Another test was carried out to determine liquid permeability of the samples by determining the speed of wetting, as described in Iverson et al., 2007. As our samples look totally mat black we do not need a pigment to see the front of wetting. It is clearly seen on natural light. Our tests with deionized water and a stopwatch show that the speed of wetting of sample A is $0.9 \mathrm{~cm}$ per second and for sample B $1.1 \mathrm{~cm}$ per second. As this method is not accurate enough we assume that both samples have a wetting speed of around $1 \mathrm{~cm}$ per second.

\section{CONCLUSIONS}

From the experimental results it can be concluded that the procedure used can create wicks with desired porosity and pore radius. These features can be created quickly, economically, at room temperature, and at relatively low price. The wicks are on the base of electrochemically produced dendrite structure. High density current stage produces powder like coatings with low mechanical stability.

Second stage of low current density stabilizes mechanically the coatings. This is achieved without any optimization of the current density and salt concentration. Our results show that the used method of deposition can be applied to produce heat pipe wicks that employ water as heat transfer medium.

A new method to make the pure copper hydrophilic was successfully developed and tested. Experimental tests for porosity (64\% for sample A and $83 \%$ for sample B) and liquid permeability (of $1 \mathrm{~cm} / \mathrm{sec}$ ) show a very good behavior of dendrite structure created as a heat pipe wick. A number of tests were carried out to compare water evaporation capabilities of samples with different depth of the wick (sample A $250 \mu \mathrm{m}$ and sample B 500 $\mu \mathrm{m}$ ) and determine the dissipated heat power. In our test conditions the ticker sample (B) behaves significantly better.

For the case when the thermal conductivity of the studied structures is not known, we recommend the described method of electrodeposition for wicks as applicable for low and medium heat flux density heat pipes.

\section{REFERENCES}

P.D. Dunn, D.A. Reay, 1978, Heat Pipes, second ed., Pergamon Press, Oxford, England

Mohamed S. El-Genk, Amir F. Ali, 2010, "Enhanced nucleate boiling on copper micro-porous surfaces", International Journal of Multiphase Flow, 36, 780-792

http://dx.doi.org/10.1016/j.ijmultiphaseflow.2010.06.003

A. Faghri, Heat Pipe Science and Technology, Taylor and Francis, Washington, DC, 1995.

Brian D. Iverson, Tyler W. Davis, Suresh V. Garimella, 2007, "Heat and mass transport in heat pipe wick structures," Journal of

Thermophysics and Heat Transfer, 21(2), 392-404

Jeong-Han Kim, Ryoung-Hee Kim, Hyuk-Sang Kwon, 2008, "Preparation of copper foam with 3-dimensionally interconnected spherical pore network by electrodeposition", Electrochemistry Communications, 10,1148-1151 http://dx.doi.org/10.1016/j.elecom.2008.05.035

Jingchun Min, Ralph L. Webb, 2002, "Long-term wetting and corrosion characteristics of hot water treated aluminum and copper fin stocks," International Journal of Refrigeration, 25, 1054-1061 http://dx.doi.org/10.1016/S0140-7007(02)00010-5

N.D. Nikolić, K.I. Popov, Lj.J. Pavlović, M.G. Pavlović, 2006, "Morphologies of copper deposits obtained by the electrodeposition at high overpotentials," Surface \& Coatings Technology, 201, 560 566

http://dx.doi.org/10.1016/j.surfcoat.2005.12.004

G.P. Peterson, 1994, An Introduction to Heat Pipes: Modeling, Testing, and Applications, John Wiley, New York 